\title{
Disrupting attention: The need for retrieval cues in working memory theories
}

\author{
DOUGLAS L. NELSON and LEILANI B. GOODMON \\ University of South Florida, Tampa, Florida
}

\begin{abstract}
Sometimes in conversation, something is said that causes us to want to comment, but before our impending but implicit thoughts can be expressed, the conversation is disrupted. Later, we cannot recall what we wanted to say, but still later, we can. We used the extralist cuing task to model this phenomenon, and across experiments we varied the strength, direction, and directness of the relationship between the retrieval cues and the targeted information. Disruption was varied by switching attention to a different task before testing and by changing the testing context. Such disruptions reduced recall for the target and its implicitly activated memories. Following a disruption, stronger cues that were related to the target or to both the target and its implicit memories were more effective than those that were related to implicit memories. The findings were consistent with a model of long-term working memory that attributes forgetting to a loss of access to what has been activated, which loss is relative to the strength of the retrieval cue. Decay alone does not explain the results, indicating that many models of working memory need to be revised to take the nature of retrieval cues into account.
\end{abstract}

In conversation, something is sometimes said that makes us want to interject a comment lying in the shadows of awareness, but before we can express it our thoughts get disrupted. The conversation may suddenly shift, someone new may join the group, and so on. The conceptual focus of attention is shifted, and the result is that an imminent comment often cannot be recovered after the disruption has passed. We often cannot revive the thought even when it is important and even when the people and the place have not changed. Later, we sometimes recall what we wanted to say because some cue helps revive the conversation and the unspoken comment. In this paper, we present a paradigm and a theoretical analysis of how disruptions of attention cause implicitly activated memories to be forgotten. As is suggested in Figure 1, the theoretical analysis is based on the idea that an episode implicitly activates related memories that can serve as the basis for a comment. Two important questions are (1) what cues revive such memories and (2) how do they work?

If attentional shifts alone cause forgetting, we may need a new theory of forgetting. The experience of this type of memory failure suggests that its cause is the disruption of attention, not decay or interference. Decay theory attributes forgetting to disuse, or to lost activation (Thorndike,

This research was supported by Grant MH16360 from the National Institute of Mental Health to D.L.N. The authors thank Cathy McEvoy for her helpful suggestions about this paper and Erin Cotrone for collecting the data for Experiment 3. Correspondence concerning this article should be addressed to D. L. Nelson, Department of Psychology, University of South Florida, Tampa, FL 33620-8200 (e-mail: dnelson2 @ chumal.cas.usf.edu).
1914), but this attribution seems incorrect, because rumination often brings the thought to consciousness. Once decayed, how could a thought be recovered? Furthermore, such thoughts appear to be brought to consciousness by some cues or events, but not by others. If decay is the only factor, one type of test cue should be as good as another. Traditional interference theory attributes forgetting to competition between similar responses acquired before or after the episode (see, e.g., McGeoch, 1942). Because competition among related memories is likely to be high on an immediate test, and because such competition can decline over delays, decay theory can explain why an implicitly activated memory can be lost and then recovered. However, the theory is mute about the relationship between disruptions of attention and implicit memories. Just as importantly, studying associatively related words in successive lists does not differentially impair the recall of implicitly activated memories in comparison with studying lists of unrelated words (Nelson, McEvoy, Janczura, \& Xu, 1993). Specific interference effects in the standard retroactive interference paradigm are not found.

Theories of working memory are relevant to this issue, because they assume a close relationship between attention and memory (see, e.g., Baddeley, 2002; Cowan, 1988, 1999; Engle, Tuholski, Laughlin, \& Conway, 1999; Ericsson \& Kintsch, 1995; Just \& Carpenter, 1992; Schneider, 1999). In these theories, working memory generally refers to attentional control processes that maintain information in an accessible state. The theories are relevant to the forgetting of implicitly activated memories, because they imply that switching the focus of attention causes the decay of activated information (see, e.g., Baddeley, 2002; 


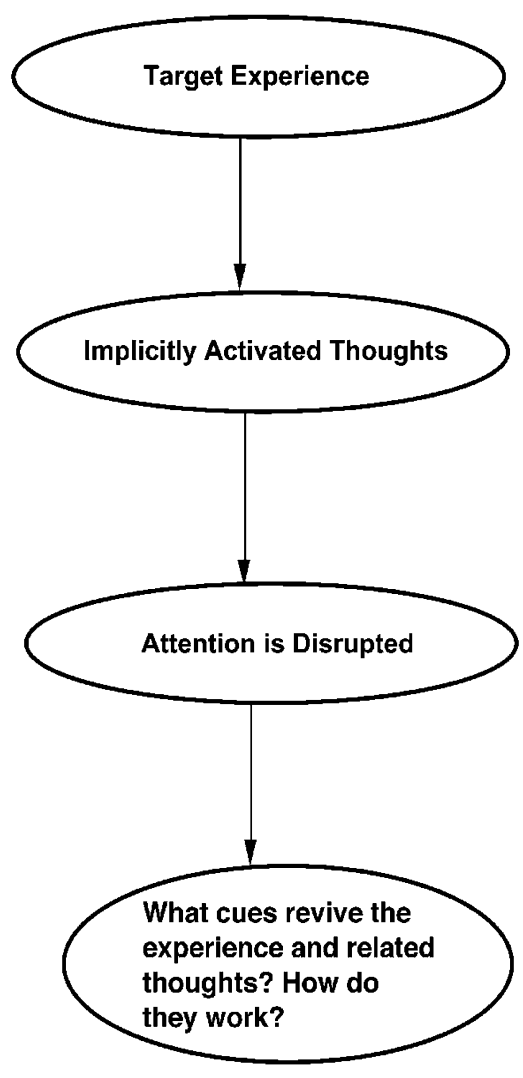

Figure 1. Episodic experience implicitly activates related memories. Disruptions of attention cause these memories to be forgotten, but later they can be remembered. Two important questions are (1) what types of cues revive such memories and (2) how do they work?

Cowan, 1999) or places limits on its access (see, e.g., Lovett, Reder, \& Lebiere, 1999; Nelson, McKinney, Gee, $\&$ Janczura, 1998). The models that attribute the loss to decay alone explain why a disruption can cause the loss of a memory that is outside the focus of attention, but whether they explain recovery is unclear. Cues can point to longterm memory representations in such models (see, e.g., Cowan, 1999), but how such cues help retrieve the needed information and how they overcome the entropy induced by decay is unclear. These theories provide a useful starting point as explanations for how interruptions of attention can cause memory to fail, but they have not been developed to a level at which they could predict how cues help recover what apparently has been lost.

\section{A paradigm and an alternative model of working memory}

In addition to a theory of working memory, what is needed is a model of cued recall and a paradigm that speak to the question of how cues help recover direct experiences as well as the related memories that they activate. In this article, we use a cuing paradigm and a model of recall called PIER2 (Processing Implicit and Explicit Represen- stations) to explore this issue (Nelson et al., 1998). PIER2 is a theory about long-term working memory that is focused on explaining the effects of implicitly activated memories in episodic tasks. The theory is primarily constructed around the extralist cuing task. Words called targets are studied, and then the recall of these targets is prompted by associatively related words serving as test cues. We use this task because words implicitly activate related words, as MAGAZINE may implicitly activate book, read, article, and so on (Underwood, 1965). Experiencing a word automatically activates its past associative history, because comprehension requires access to past as well as to present experience (Kintsch, 1988). We also use this task because words serving as retrieval cues are not a part of the study list and can be selected so as to be related to the studied words, to their implicitly activated memories, or to both. With the studied word serving analogously as the target experience to be recalled, and its associates analogously serving as implicitly activated memories, the extralist cuing task provides a model for investigating how shifts of attention disrupt implicitly activated memories and how cues help recover such memories. For example, in mulling over a conversation, one is more likely to remember that what was forgotten was a point about a new magazine after receiving a notice that a book order is overdue. The cue book activates related memories, including magazine, as well as memories related to these activated memories.

Work with this paradigm has shown that recall varies with past associative experience with the words that serve as targets and as test cues (see, e.g., Nelson et al., 1998). Some words implicitly activate smaller sets of associated words, and such words make more recallable targets and better cues. Words also differ in the density of the connections among their associates, or what is called connectivity. Greater connectivity among the associates of a studied word promotes higher levels of recall and recognition. Both effects are related to implicitly activated memories, and disrupting attention to the encoding episode reduces such effects (see, e.g., Nelson et al., 1993; Nelson et al., 1998). In a task requiring multiplication of numbers immediately after a word list is seen, recall declines and the relative recall advantages for words of small set size or high connectivity are lost. Such losses are the result of attention switches rather than of interference, because studying other lists of either similar or dissimilar words during a comparable interval reduces recall, but has no influence on the magnitude of set size effects (Nelson et al., 1993).

PIER2 assumes that disruptions reduce target set size and connectivity effects because they reduce access to what the target activated in long-term working memory during encoding. A weak cue that normally would have worked now fails, but when stronger cues are presented after the math task, full set size and connectivity effects are found (Nelson et al., 1998). Moreover, set size effects reappear for weak cues when a second list of words is studied after the math task (Nelson et al., 1993). Studying another list after the disruption apparently reinstates the 
encoding context strongly enough for target set size effects associated with the original list to reemerge. Neither decay theory nor interference theory can explain such results.

\section{The importance of linking connections}

Existing findings suggest that a word's implicitly activated associates will affect its recall as long as attention is maintained on the episode or, in the case of distraction, is redirected to the episode by a strong cue or by a weak cue plus a similar encoding experience in the same context. The contribution of implicitly activated memories to retrieval is apparently affected by attention switching and by retrieval cues, both specific and context related. These findings, however, have been limited to manipulations of memories activated by the target, such as set size and connectivity.

The specific purpose of this research is to determine whether attention disruption reduces the effects of preexisting connections that link the cue with the target. Linking connections define the cue-target relationship and vary in strength, direction, and directness (Nelson et al., 1998). Strength is indexed by the probability that one word will be given as a response to another in free association, with more probable words being more strongly related (Nelson, McEvoy, \& Schreiber, 1999). Connections are directional in that they can run from cue to target (forward links) or from target to cue (backward links). Finally, connections can be direct, as when one word directly produces another as an associate, or they can be indirect, as when two words are related through other words. Mediated connections involve associated words that intervene between the cue and the target-for example, DAISY-flower-ROSE. Shared associate connections occur when the cue and the target produce a common associate-for example, the cue DAISY and the target ROSE both produce flower.

On a nondisrupted test, all four types of linking connections affect recall in the extralist cuing task (Nelson \& Zhang, 2000). Recall is higher for stronger than for weaker connections, and for forward than for backward connections. It is also higher when indirect connections are available. The evidence suggests that the recall of a recent experience is best described as the net result of computing the connections that link the cue and the target relative to associates that are unique to the cue or to the target (Nelson et al., 1998). Unique or nonlinking associates are correlated with a word's set size and drive recall downward. For example, BOOK may be a good cue for recalling MAGAZINE, because these words are directly associated in both forward and backward directions and because they are linked by four mediators and three shared associates. However, the effectiveness of вОок as a test cue will be reduced, because it produces unique associates that are not linked to MAGAZINE, such as worm, and because MAGAZINE produces unique associates, such as Vogue. More generally, the effectiveness of any retrieval cue in prompting the recall of a recent experience is a net result of computing the pool of connections that link the items relative to the noise produced by competing associates (Nelson et al., 1998).

In Experiment 1, we studied the effectiveness of direct forward and backward connections in prompting recall before and after attention was disrupted. Participants studied a list of target words and were tested with retrieval cues that held forward (F), backward (B), or forward + backward $(\mathrm{F}+\mathrm{B})$ links with their targets. Forward-linking cues are directly related to their targets in long-term memory, and backward-linking cues are directly related to one of their target's implicitly activated memories. $\mathrm{F}+\mathrm{B}$ cues are related to both the target and one of its implicitly activated associates. A control with no connections in either direction was not included, because recall with such a list is near zero even in the absence of disruption (Nelson et al., 1998). Different groups were tested without disruption, after a 20 -min disruption produced by a multiplication task, and after 20-min of multiplication plus a 24-hr delay. In Experiment 2, we studied the influence of switching attention to the math task on mediated and sharedassociate connections. In Experiment 3, we studied the effects of a different type of disruption. Instead of switching conceptual attention between study and test, we disrupted context by moving the testing session to a different room.

\section{EXPERIMENT 1}

PIER2 predicts that the effects of both direct and indirect links will be susceptible to disruptions produced by switching attention to conceptually different tasks. The model assumes that, during encoding, experiencing a familiar word implicitly activates its representation and the representations of related words in long-term working memory. Preexisting links among these words and from these words to the target increase the activation levels of the target and its associated words. In the model, these activation levels increase net cue-target strength and the probability of sampling the target. For nondisrupted tests, the model predicts that cues with $\mathrm{F}+\mathrm{B}$ links will produce greater recall than cues with either F links or B links. Adding another source of strength to the relationship increases net cue-target strength. In addition, the model predicts that cues with $\mathrm{F}$ links will generally produce higher recall than will those with B links. The Appendix presents a computational example of how the model computes the probability of recovering the target on a nondisrupted test.

More importantly for the present purposes, the model predicts that the ordering of these cues will be maintained on disrupted tests as long as the probability of accessing the target from the cue remains above the baseline defined by free association. Forward free-association strength determines the probability that the target will be produced in the absence of a study trial, and it represents the lower boundary on recall. Hence, disrupting attention reduces access to what was activated during study, but this loss is not absolute. The model assumes that access is relative to the net strength of the cue-target relationship. As access 
probability declines, losses in access to what has been activated in long-term memory will be reduced in the presence of a test cue with a stronger preexisting relationship with its target. The model makes specific predictions about net cue-target strength, but it does not predict the time course of access losses produced by disruption, because this aspect of the model has not been developed. Nevertheless, it does predict that the probability of recall after a disruption will approach the free-association probabilities of .13 for $\mathrm{F}+\mathrm{B}$ and $\mathrm{F}$ links, and zero for $\mathrm{B}$ links.

\section{Method}

Design and Participants. The experimental design formed a $3 \times 3$ between-subjects factorial. Test cues had F+B, F, or B links with their targets. In the different conditions, testing was either not disrupted, was disrupted by $20 \mathrm{~min}$ of multiplication, or was disrupted by $20 \mathrm{~min}$ of multiplication plus a $24-\mathrm{h}$ delay. A total of 180 participants took part in the study, with 20 assigned to each of 9 between-subjects conditions. In each condition, 10 participants were assigned to one of two lists and 10 to the other. All were undergraduates taking psychology courses who received extra credit for participating, and they were randomly assigned in replication blocks to conditions and lists.

Materials. Two lists, each consisting of 24 targets and three cues per target, were constructed by selecting items from free-association norms (see Appendix C in Nelson et al., 1998). The targets (e.g., CLOSET) were held constant, and the manipulations of forward and backward connections were implemented by varying the test cues. One cue for each target had bidirectional links with it (e.g., HANGER), one was connected with it in the forward direction (e.g., BROOM), and one was connected with it in the backward direction (e.g., DOOR). Regardless of direction, connection strength averaged $.13(S D=.06)$ according to free-association norms (Nelson et al., 1999). When either a forward or backward connection was manipulated, strength in the opposite direction was weak, averaging zero in the norms. These differences mean that, in the absence of a study experience, approximately 20 out of 150 participants produced the target in free association for strong links, and none produced it for weak links.

Other variables known to affect extralist cued recall were held constant. Test cues shared an average of 1.42 associates $(S D=1.07)$ and .95 mediated connections $(S D=.95)$ with their targets. All pairs were about equally and weakly related in terms of indirect connections regardless of the values of the direct connections. Cue and target set size averaged 13.87 associates $(S D=4.85)$ and 14.50 associates $(S D=4.41)$, respectively. Target frequency values averaged 79 words per million $(S D=107)$, and the mean numbers of connections to the targets from their associates and the mean numbers of connections among each target's associates averaged $6.15(S D=3.12)$ and $1.41(S D=0.64)$, respectively.

Procedure. All data were collected in individual sessions. During the study trial, the participants were asked to read each word aloud when it was shown and to try to remember as many words as possible, without being told how they would be tested. Each study word was presented for $3 \mathrm{sec}$ on a computer monitor, and prior to the study trial, a short list of names was shown to familiarize them with this rate.

Immediately following the last study word, the participants either were given the instructions for the test trial or started on the disruption task. Those in the nondisrupted condition were read the test instructions, which told them that cue words were going to be shown one at a time and that each cue would be meaningfully related to one of the studied words. They were asked to read each cue aloud and then try to recall the target word that went with it, with guessing allowed but not required. The participants in the disrupted conditions were given sheets containing multiplication problems (e.g., $278 \times$
$393,45 \times 265$, etc.). They were asked to solve as many problems as they could, as quickly and as accurately as they could. One group worked for 20 min on this task, and then was given the cued-recall instructions. Another worked for $20 \mathrm{~min}$, was dismissed and asked to return $24 \mathrm{~h}$ later, and then was given the test instructions. The test trial was self-paced, and the orders of appearance of both study and test words were independently and unsystematically randomized for each participant.

\section{Results and Discussion}

The results are displayed in Figure 2, which shows probability of correct cued recall as a function of direction and disruption, and, as can be seen, recall varied with both variables. When attention was not disrupted, recall was highest when the cue-target pairs were joined by $\mathrm{F}+\mathrm{B}$ links, was next highest when they were joined by $\mathrm{F}$ links, and was lowest when they were connected through B links. When attention was disrupted, recall declined with the length of the disruption. However, although the relative ordering of the three types of cues was the same after the 24-h delay, the differences among the cues were reduced. This finding suggests that the probability of accessing the target from these cues was beginning to approach the freeassociation baseline defined by forward strength.

The results of an analysis of variance (ANOVA) were consistent with this description. The effects of direction were significant $\left[F(2,171)=99.12, M S_{\mathrm{e}}=0.009\right]$, as were the effects of disruption $[F(2,171)=51.26]$. Recall was highest in the $\mathrm{F}+\mathrm{B}$ condition (.42), next highest in the $\mathrm{F}$ condition (.28), and lowest in the B condition (.18). Recall was highest when attention was not disrupted (.38), next highest after a 20-min disruption (.28), and lowest after a 24-h disruption (.21). A Fisher's two-tailed least significant difference (LSD) for planned comparisons among each set of means was .03. Importantly, the interaction between direction and disruption was also significant $[F(4,171)=5.05, \mathrm{LSD}=.06]$. The differences among the three types of test cues were significant in each disruption condition, but they tended to be smaller after the 24-h disruption than on the immediate test. Recall for B links dropped to a low level after 20 min of multiplication and remained at that level at the 24-h delay. These levels of recall, however, were significantly above chance. Test cues having only B links did not produce their targets in free association, and $t$ tests for single means showed that recall was significantly greater than zero at $20 \min (t=12.20)$ and $24 \mathrm{~h}(t=7.49)$. Note that, although B links contributed to recall after $24 \mathrm{~h}$, this contribution was most evident when $\mathrm{F}$ links were also present.

The results of Experiment 1 indicate that switching attention from a conceptually demanding memory task to a conceptually demanding problem-solving task reduces the benefits provided by cues with connections to the targeted information. As with target set size and connectivity effects, the retrieval benefits produced by cues having preexisting connections to the target are reduced when the focus of attention on a particular memory task is disrupted by the need to focus on something else. Such results are consistent with current models of working memory that 


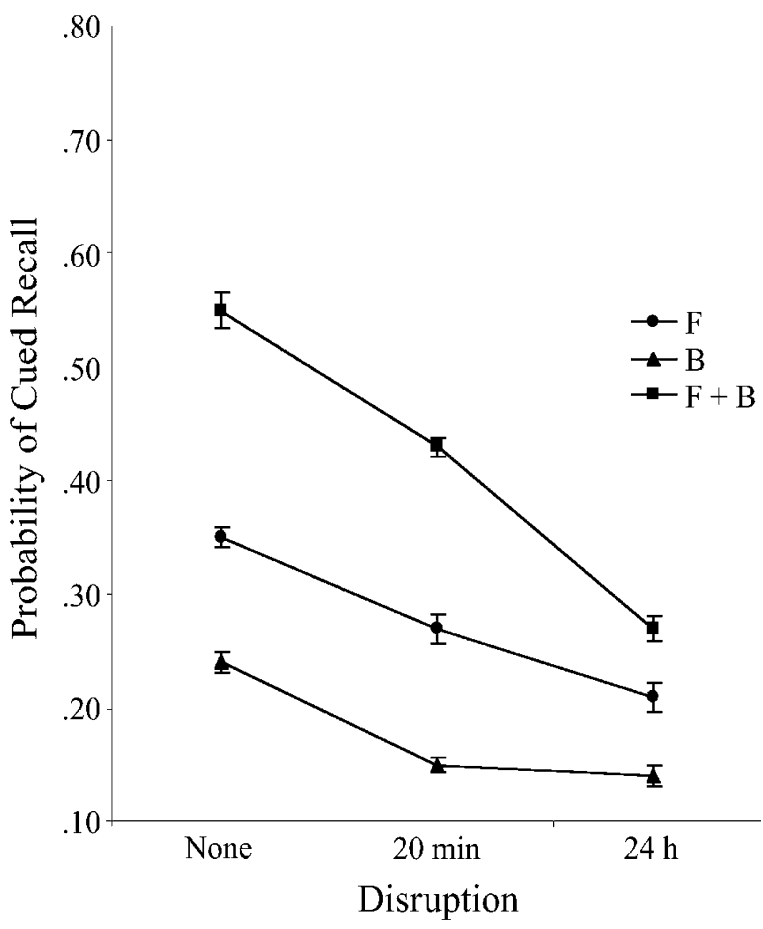

Figure 2. Probability of cued recall as a function of type of test cue and disruption. Test cues were linked to their targets in a forward $(F)$ or back ward $(B)$ direction, or in both directions $(F+B)$. The error bars represent standard errors of the mean in this and in the remaining figures.

predict that activation decays unless attention is maintained (e.g., Cowan, 1999). However, they go further in showing that the deleterious effects of that decay are related to the strength of the linking connections joining the cue-target pairs. In PIER 2, adding an F link to a B link produces greater net cue-target strength. As with set size and connectivity effects, a stronger cue reduces the impact of disruptions.

In PIER2, disruption reduces cue-target strength because the primed activation levels of the target and its associates become less accessible in long-term working memory (Nelson \& Goodmon, 2002). Test cues can be connected to many associates besides the target and, in practical terms, normatively stronger associates of the test cue are more likely to be sampled than the target as accessibility declines. If this analysis is correct, the proportion of intrusions that are stronger than the target in terms of freeassociation norms will vary with the disruption manipulation. Intrusions should also vary with the direction of the linking connections, because the opportunity for such intrusions covaries with direction. Because B-linked test cues do not produce the target as an associate, virtually all of the cue's associates are stronger than the target. As is shown in Table 1, these expectations were confirmed. The table shows the mean proportions of intrusions that were stronger than the target relative to the total number of intrusions, calculated for each participant. Intrusions varied with direction $\left[F(2,171)=77.07, M S_{\mathrm{e}}=0.022\right]$ and disruption $[F(2,171)=11.73]$, and the interaction was unreliable $(F<1)$. Mean intrusions were $.40, .38$, and .68 for the $\mathrm{F}+\mathrm{B}, \mathrm{F}$, and $\mathrm{B}$ directions, respectively. For the nondisrupted, 20-min, and 24-h conditions, the means were $.41, .52$, and .53 , respectively. As was expected, disrupting attention to the memory task significantly increased the probability of response from the cue's associative set that was stronger than the target.

\section{EXPERIMENT 2}

Experiment 2 was motivated by the rationale underlying the first experiment, but indirect instead of direct cue-target links were investigated. Cue-target pairs had weak forward links with their targets, along with either shared-associate + mediated links $(\mathrm{S}+\mathrm{M})$, shared-associate links (S), mediated links (M), or no indirect links (control). Recall was evaluated in the absence of disruption and after 10 and 20 min of multiplication. The question is whether disruptions differentially reduce the effectiveness of indirect links in prompting target recovery. As is shown in the Appendix, $\mathrm{S}$ links and $\mathrm{M}$ links add to net cue-target strength (Equation A2). The availability of $\mathrm{S}+\mathrm{M}$ links produces a stronger cue-target relationship than does that of either $\mathrm{S}$ links or $\mathrm{M}$ links. Hence, the model predicts that recall on nondisrupted tests will be ordered as $\mathrm{S}+\mathrm{M}>\mathrm{S}=$ $\mathrm{M}>$ no indirect. This ordering will be maintained after disruption, until the probability of accessing the implicit representation of the target and its associates begins to approach the free-association baseline. At this undefined point, the benefits of indirect links will begin to diminish, and this decline will be more apparent for weaker S links and $\mathrm{M}$ links than for $\mathrm{S}+\mathrm{M}$ links.

\section{Method}

Design and Participants. The experimental design formed a $3 \times$ $2 \times 2$ mixed-model factorial, with disruption (none, $10 \mathrm{~min}$ of multiplication, and $20 \mathrm{~min}$ of multiplication) varied between subjects. The availability of shared links (present, absent) or mediated links (present, absent) was varied within subjects. A total of 90 participants took part in the study, with 30 assigned to each of the 3 disruption conditions. In each condition, 15 participants were assigned to one of two lists and 15 to the other. All received extra credit for their service and were randomly assigned in replication blocks to conditions and lists.

Materials. Two 24-word lists were constructed by selecting items from the association norms (see Appendix D in Nelson et al., 1998). When shared associate links were available, they were strong and averaged $.20(S D=.07)$, and when mediated links were available,

Table 1

Proportions of Intrusions That Were Stronger Than the Target in the Test Cues' Sets

Direction of

Test Cue-Target Relationship

\begin{tabular}{lccc} 
& \multicolumn{3}{c}{ Test Cue-Target Relationship } \\
\cline { 2 - 4 } Disruption Condition & $\mathrm{F}+\mathrm{B}$ & $\mathrm{F}$ & $\mathrm{B}$ \\
\hline Nondisrupted & .34 & .29 & .59 \\
20-min disruption & .41 & .45 & .69 \\
24-h disruption & .43 & .40 & .75 \\
\hline
\end{tabular}


they averaged $.21(S D=.04)$. When such links were unavailable, they were extremely weak and averaged $.003(S D=.003)$ in each condition. Other variables known to affect cued recall were equated as closely as possible within each condition, including forward strength $(.12, S D=.08)$, backward strength $(.02, S D=.03)$, cue set size $(10.56, S D=2.90)$, target set size $(13.09, S D=4.17)$, mean connectivity per associate $(1.37, S D=.67)$, frequency $(120, S D=78)$, and concreteness $(4.82, S D=1.23)$.

Procedure. The general procedure was identical to that used in Experiment 1, including the use of the extralist cuing procedure, study instructions, and rates of study presentation and testing. However, testing was disrupted for 10 or for $20 \mathrm{~min}$.

\section{Results and Discussion}

The results of Experiment 2 are displayed in Figure 3. When there was no disruption, target recall was highest for S+M links, next highest for S links or M links, and lowest when neither type of indirect link was available. This relative ordering was maintained after $10 \mathrm{~min}$ of multiplication, although a slight advantage for mediated over shared-associate links began to emerge. After 20 min of multiplication, the pattern changed. The relative recall advantage found when both types of indirect links were available was maintained, but the advantages of having only a single indirect link were lost for shared associates and, to a slightly lesser extent, for mediators.

Attention and implicit memory. The results of a mixed-model ANOVA indicated that the probability of cued recall varied with disruption $[F(2,87)=133.49$, $\left.M S_{\mathrm{e}}=0.022\right]$, mediated connections $[F(1,87)=71.16$,
$\left.M S_{\mathrm{e}}=0.016\right]$, and shared-associate connections $[F(1,87)=$ $\left.47.34, M S_{\mathrm{e}}=0.016\right]$. Highest recall was found in the absence of disruption (.61), the next highest after the 10-min disruption (.48), and the lowest after the 20-min disruption (.30). An LSD of .04 indicated that all three means were different from each other. Higher recall was also found when shared associates were available (.51) than when they were not (.42), and when mediators were available (.52) than when they were not (.41).

Of the interactions, only the disruption $X$ mediator $X$ shared-associate interaction was reliable $[F(1,87)=8.05$, $M S_{\mathrm{e}}=0.015$; Fisher's LSD $\left.=.06\right]$. On the nondisrupted test, recall was ordered in terms of net cue-target strength as expected: $\mathrm{S}+\mathrm{M}$ connections $>\mathrm{S}=\mathrm{M}$ connections $>$ no indirect connections. All differences were significant. This pattern was also apparent after a 10-min disruption. In contrast, after 20 min of disruption, the advantage of having both types of indirect links was still apparent, but the advantages of having only a single indirect link were essentially eliminated. Recall was higher when both indirect links were available (.43) than when mediators (.28), shared associates (.24), or no indirect links (.24) were available. A separate ANOVA on the 20-min data indicated that mediated connections $\left[F(1,29)=18.27, M S_{\mathrm{e}}=\right.$ $0.010]$, shared associates $[F(1,29)=27.20]$, and the interaction of these two sources $[F(1,29)=19.10, \mathrm{LSD}=.05]$ were significant. After $20 \mathrm{~min}$ of attention to the math task, shared associates and mediators affected recall probability only when both links were available.

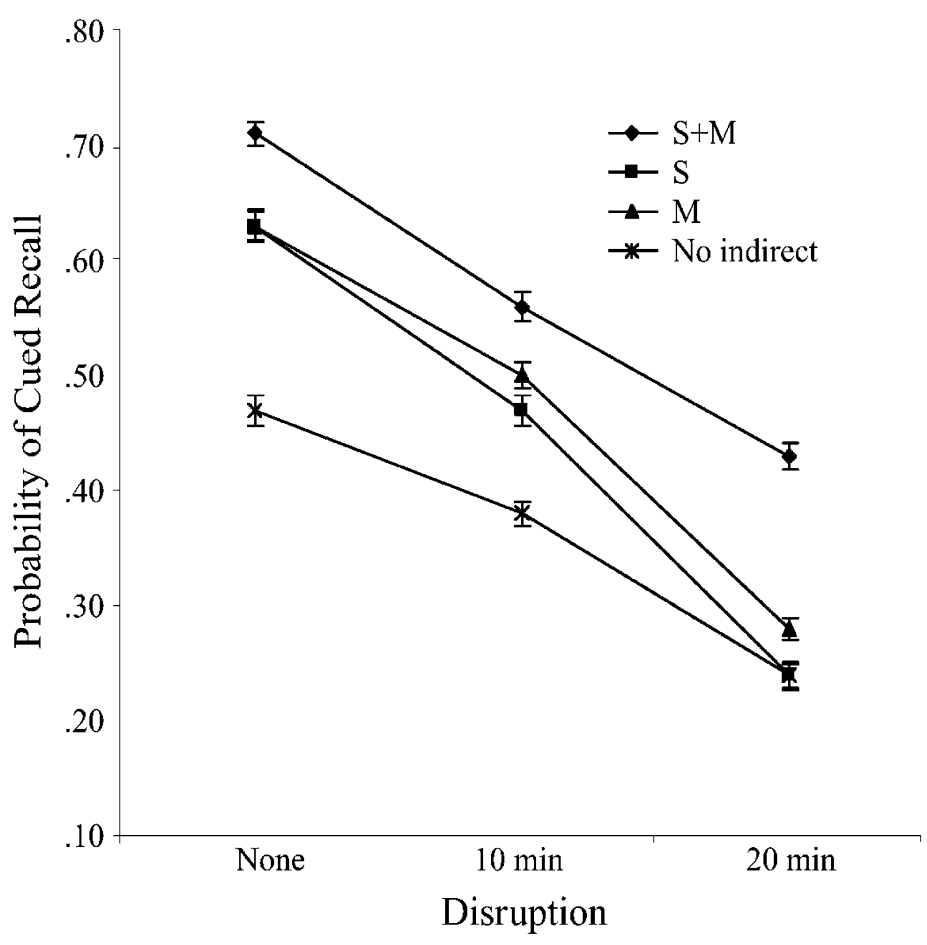

Figure 3. Probability of cued recall as a function of type of test cue and disruption. All test cues were linked to their targets in the forward direction and, in addition, they could share associates (S) or mediators (M). 
The results of Experiment 2 indicate that indirect links add their effects to direct links in cued recall, and that these effects are reduced when attention is shifted away from the memory task to a multiplication task. After the participants had spent 20 min multiplying numbers, their recall based on a single type of indirect link did not differ significantly from the control, in which neither link was available. In contrast, recall based on the availability of both mediators and shared associates remained significantly above that based on the control. The beneficial effects of shared associates are apparent even after a relatively lengthy disruption when mediated links are added to the cue-target relationship. Similarly, the beneficial effects of mediators are apparent after the disruption when shared associates are added to the relationship. In PIER2, adding indirect links of either type strengthens the net cue-target relationship and increases the odds of target recovery. As with forward and backward links, mediators and shared associates facilitate recall after an extended disruption as long as a strong cue is used to recover the memory. A stronger cue-target relationship is more likely to revive both an interrupted target experience and what it implicitly activated.

According to PIER2, diverting the focus of attention to a different and demanding cognitive task reduces longterm working memory access to the target and to its associates. This information becomes less accessible with more extensive disruptions, but the loss depends on the confluence of the activated information and the information supplied by the test cue. Weakly related test cues that were effective in the absence of a disruption are less able to revive what was primed during the original experience. Forward cue-to-target links and mediators are less effective because the primed representation of the target becomes less accessible, and backward target-to-cue links and shared associates are less effective because the primed associates of the target become less accessible. However, the results of Experiments 1 and 2, as well as earlier findings (Nelson et al., 1993), indicate that stronger cues are more likely to revive the implicit representation. The recovery of an implicitly activated memory depends on access probabilities, but the findings indicate that it also depends on net cue-target strength. Recovery of an implicitly activated memory appears to be best described as the result of computing the accessibility of the target and its associates in relation to the test cue and its associates. Access in working memory plays a role, but it is a relative role that is determined by the strength of the test cue in relation to the accessibility of the target and what it implicitly activated during learning.

\section{EXPERIMENT 3}

Earlier findings indicated that target set size effects are revived by weak cues after a disruption, provided that a new list of words is studied just prior to testing. It did not appear to matter whether or not the words in the new list were associatively related to the words of the original list
(Nelson et al., 1993). This finding suggests that studying a list of words after a disruption can have the effect of reinstating contextual cues bound to the original encoding experience. Once context cues have been reinstated, weak cues can recover the target and reactivate its associates. The purpose of Experiment 3 was to investigate the effects of a change of test context on the influence of $F$ links and B links. Participants studied the targets in one room, and were tested either in the same room or in a different room. Changes in test context are known to reduce recall (see, e.g., Smith, 1979), and the question was whether changing rooms would have a differential influence on the ability of $\mathrm{F}$ links and $\mathrm{B}$ links to revive the target.

PIER2 does not make a clear prediction about the outcome of this experiment. On one hand, the model predicts that context disruptions will have a more detrimental effect on B links because, as Experiment 1 shows, they were weaker than F links. As net cue-target strength decreases, context cues become differentially important. On the other hand, the model predicts that $\mathrm{F}$ links are more dependent on context cues than are B links. The model assumes that studying the target produces an explicit as well as an implicit representation, and that the explicit representation directly incorporates contextual cues (Nelson et al., 1998). Target words are seen, named, and possibly rehearsed with other list words, and these mental operations take place in a specific context. When these context cues are more readily available during testing, targets will be more accessible to cues that normally produce them as associates (Nelson \& Goodmon, 2002). Cues with F links to the targets normally produce them in free association, whereas cues with only B links do not. Hence, F links may be more vulnerable to disruptions produced by changing the testing context. These two effects work against each other, and the model provides no means at present for eliminating this ambiguity. We present this experiment as the first empirical study of the effects of context on extralist cued recall.

\section{Method}

Design and Participants. The experimental design formed a $2 \times 2$ between-subjects factorial. Link direction (forward, backward) was crossed with testing context (same, different). Twenty participants were assigned to each condition, 10 to each list.

Materials and Procedure. The forward and backward lists used in Experiment 1 were also used in the present experiment. Except for the study instructions, the study procedures were also the same. Words were shown one at a time in single study sessions, and they had to be read aloud when shown. Instead of receiving intentional learning instructions, the participants were asked to rate each study word for concreteness on a scale of 1-5. The only known test difference was that the experiment took place in two different labs on the same floor of the building. Half of the participants studied the list in one lab and were walked to the other lab to be tested. One lab was a single room, and the other was comprised of a larger room surrounded by six smaller testing rooms. Otherwise, the two rooms were similar in terms of computers and furniture. The walk took about $80-90 \mathrm{sec}$. The experimenter told the participants that the test had to be taken in another room and did not engage in small talk on the way. Incidental instead of intentional learning instructions were used, on the assumption that such instructions would discourage rehearsal during this short interval. Once the participants were seated 
in the testing room, the test instructions for the self-paced cued recall test were read and the evaluation was conducted in the usual way. The remaining half of the participants both studied and were tested in either the smaller or the larger lab. Immediately after the last item was shown, the experimenter turned the computer away from the participant, ostensibly to prepare the test trial, and after $80-90 \mathrm{sec}$, the computer was turned back around, and the test instructions were read.

\section{Results and Discussion}

The results of Experiment 3 are displayed in Figure 4. As is shown, cues with forward links to the target produced higher recall than did cues with backward links, and disrupting the test context reduced recall for both types of cues. The results of a between-subjects ANOVA indicated that link direction $\left[F(1,76)=34.23, M S_{\mathrm{e}}=0.012\right]$ and disruption $[F(1,76)=9.19]$ had significant effects. The mean probabilities of correct recall for forward and backward cues were .38 and .24 , respectively. For unchanged and changed testing contexts, they were .35 and .28 , respectively. Simply spending about a minute walking down the hall to take the test in a different room was a sufficient disruption to reduce the effectiveness of each type of retrieval cue. The interaction was not reliable $(F<1.0)$, but a planned LSD of .07 indicated that the effects of context change were significant for $\mathrm{F}$ links, but not for B links. Cues with $\mathrm{F}$ links appear to benefit somewhat more than do cues with B links from constantly available context cues. More work is needed, but this finding suggests that context cues play a differential role in supplementing the effects of semantically specific cues. From the perspective of recovering implicit memories after a disruption, effects produced by $\mathrm{F}$ links apparently rely more on context cues than B links do.

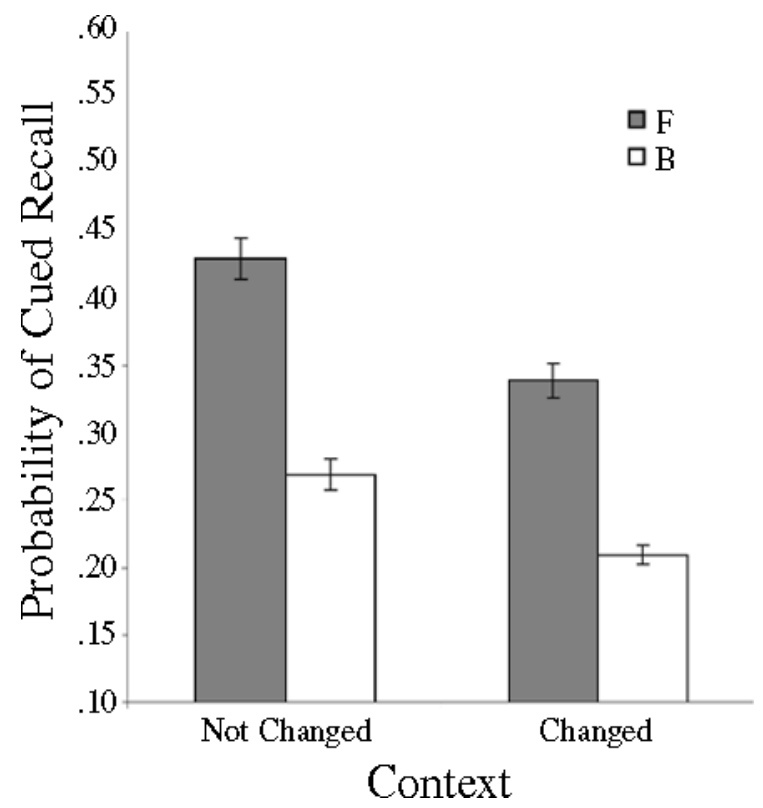

Figure 4. Probability of cued recall as a function of type of test cue and test context.

\section{GENERAL DISCUSSION}

We began this paper by noting that implicitly activated memories are susceptible to momentary disruptions of attention. After a disruption, such memories are sometimes difficult to recover moments later, even in the same physical context. Although we may be speaking with the same people in the same place, we may be frustrated because something that we perceive as needing to be said cannot be recalled on demand, despite its imminent presence prior to the interruption. Some time later, and often in a different context, the information comes to mind. The question is, how does this happen?

Our tentative answer to this question arose in the context of research on extralist cuing. Early findings indicated that the associates of a studied word are activated but have a reduced effect on its recall after attention to the memory task has been disrupted by an interpolated math task just after study (e.g., Nelson et al, 1993; Nelson et al., 1998). Target words having smaller associative sets or more connected associates no longer show a recall advantage when cued by relatively weak cues. The present findings extend these results in showing that connections that link the cue and the target together have reduced effects when attention to the memory task has been disrupted either by a problem-solving task or by simply changing rooms for testing. The effects of forward (cue-to-target) links and backward (target-to-cue) links are reduced, as are the effects of mediated and shared associate links. In general, switching attention to a different conceptual task or to a different testing context is sufficient to produce forgetting of explicitly encoded and implicitly activated information. Interruptions reduce our ability to recall an explicit experience and its implicitly activated memories.

Most theories of working memory attribute such losses to absolute decay (e.g., Cowan, 1988). Switching attention to another task or to a different testing context causes information activated in the initial task to decay rapidly unless it is actively maintained through rehearsal, which keeps it in the focus of attention. These theories have not yet specifically addressed the role of retrieval cues and how such cues might revive implicitly activated memories after a disruption. ACT-R also assumes that decay plays a key role in activation loss, but it differs from other working memory theories (e.g., Lovett et al., 1999). ACT-R assumes that source activation is limited and must be divided among goal nodes, defined by different tasks performed $s i$ multaneously. This model can explain why disruptions reduce the influence of implicitly activated memories in the extralist cuing task, but this success requires the assumption that source activation must be split between two tasks performed successively. The model must assume that activation is sent to the production rules for both the math task and the cued-recall task. For example, if BOоK were presented as a cue for MAGAZINE, then BOOK would have to send activation to the production rules for multiplication and to the rules for cued recall. This division would presumably have to continue for some number of cues in 
order to reduce the influence of variables such as backward strength. This assumption is feasible in simultaneously performed tasks, but we find it difficult to understand why this division of activation would continue in the successively performed tasks used here. It is also difficult to anticipate how ACT-R will explain effects related to the target's associates. At this point of development, theories of working memory do not address the role of retrieval cues and how such cues revive implicitly activated memories.

In PIER2, long-term working memory losses occur when the test cue insufficiently accesses the implicit representation of the target and its associates. The model assumes that access to the primed-activation levels of the target and its associates is reduced by the extent of the disruption in relation to the net strength of the cue-target relationship. The access loss is relative to the strength of the cue used to prompt recall. In this model, working memory losses are not absolute, but relative to the power of the test cue to reactivate the target and its associates. Given weak test cues after a disruption, cued recall becomes more akin to free association, in which the cue tends to select a normatively stronger associate than the associate serving as the target word. Cues activate sets of associates, and the associate selected as the target by the experimenter is usually not the strongest associate in the set. Competition among these associates determines selection whenever one associate is singled out for recall, and after disruption, the target begins to lose its advantage relative to stronger preexisting associates in the set. In the model's computation of Equation A1 (Appendix), the primed representations of the target and its associates become less accessible after a disruption. This decline reduces the net cue-target strength computed in Equation A2, but this reduction is relative to the preexisting strength of the linking connections derived from the test cue. In Equation A2, the activation of the target and its associates is cross-multiplied by the strengths of forward, backward, and shared associates, and mediated links. Declines in target access probability directly reduce the effects of forward strength and mediators (Nelson \& Goodmon, 2002). Declines in associate access probability reduce the effects of backward links, shared associate links, target set size, and connectivity (Nelson et al., 1998). Net cue-target strength increases as an additive function for each type of link, so adding another link to the cuetarget relationship increases the probability of recall on nondisrupted tests. It also increases the probability of recall on disrupted tests, as long as the access probability remains greater than the baseline defined by the probability of producing the target in the absence of prior study.

In this model, implicitly activated information is lost because of access failure caused by a weaker cue-target intersection, not because of interference or absolute decay levels (Nelson et al., 1993). For this reason, it differs from other theories of working memory designed to explain performance in other types of tasks. In PIER2, the amount of forgetting observed depends on the net strength of the relationship between the test cue and its target. Both set size and connectivity effects survive a short disruption when a strong cue is used to prompt recall, but not when a weak cue is used (Nelson et al., 1993; Nelson et al., 1998). Similarly, as the present experiments show, the effects of both direct and indirect links are more likely to survive a disruption when the test cue also has a second, relatively strong preexisting link to its target. For example, given a strong forward link, the backward link contributed substantially more to the level of overall recall even after a 24-h disruption. In the absence of a measurable forward connection, cuing effects linked to backward connections were above chance but were especially weak after only 20 min of multiplication. Similarly, given a direct forward link bolstered by indirect mediated links to the target, shared-associate links contributed significantly to recall after a 20-min disruption. In the absence of the mediated links, the presence of strong shared-associate links failed to add anything to target accessibility beyond what was already provided by the forward connection.

In PIER2, context cues play an important role in the explicit representation of the target. ${ }^{1}$ Naming and studying the target produces an explicit, context-linked episodic representation. The explicit representation of the target adds to its accessibility as a response to any cue that normally produces it as a response. When more accessible as a result of a recent episodic encoding, a cue with a forward preexisting link to the target experience will now produce it with greater likelihood (Nelson \& Goodmon, 2002). Similarly, links involving a target's implicitly activated associates indirectly benefit from their connection to the context through the target. The target's associates, including the backward link to the test cue, are indirectly linked to context because they are bound to the target.

Disrupting context cues will therefore influence the effects produced by both the explicit and the implicit representations of the target. Context cues remain salient when the encoding and testing contexts are the same, and are disrupted when they change. The retrieval effectiveness of both forward and backward links was reduced when testing was moved to a different room. Switching places and switching mental tasks reduces the effectiveness of both forward-acting cues that normally activate the target, and backward-acting cues that are activated by the target. Interestingly, switching places and switching mental tasks would seem to involve very different mental processes, but the effects of such switches may be mediated by the same mechanism: the disruption of distinctive context cues. This disruption can be overcome by tasks similar to those performed during encoding, in a sense by ruminating about the event (Nelson et al., 1993). It can also be overcome by a particularly strong retrieval cue, as the present studies suggest, and by engaging in an intention to use whatever cues are available to recall a context-specific memory (Humphreys, Tehan, O'Shea, \& Bolland, 2000; Nelson \& Goodmon, 2002).

According to PIER2, we momentarily forget impending comments after a disruption because currently available retrieval cues-both specific language cues and context cues-fail to reactivate information that was consciously 
processed and unconsciously activated just prior to the disruption. Self-instructions to retrieve the comment and context cues alone are important, but they can be insufficient in the absence of strong specific cues that directly provide features of or prior associations to the needed information. Some time later, we may recall what we had wanted to say, but primarily because some cue conjured up during rumination or through accident has revived the conversation and the impending comment. What is needed is information that is specific enough to compute the intersection between available cues and what was activated originally, information that will get us beyond guessing on the basis of prior knowledge alone. The present findings indicate that the linking information is likely to involve many different types of links, and that what matters most for success is the outcome of a complex computation of net strength. Interestingly, because of differences in net strength, cues comprised of the implicitly activated memories of a target experience are less effective than those that normally bring the target to mind.

\section{REFERENCES}

BADDEley, A. D. (2002). Is working memory still working? American Psychologist, 3, 849-864.

Cowan, N. (1988). Evolving conceptions of memory storage, selective attention, and their mutual constraints within the human information processing system. Psychological Bulletin, 104, 163-191.

Cowan, N. (1999). An embedded-process model of working memory. In A. Miyake \& P. Shah (Eds.), Models of working memory: Mechanisms of active maintenance and executive control (pp. 62-101). Cambridge: Cambridge University Press.

Engle, R W., Tuholski, S. W., Laughlin, J. E, \& Conway, A. R. (1999). Working memory, short-term memory, and general fluid intelligence: A latent-variable approach. Journal of Experimental Psychology: General, 128, 309-331.

ERICSSON, K. A., \& KinTsCH, W. (1995). Long-term working memory. Psychological Review, 102, 211-245.

Humphreys, M. S., Tehan, G., O'Shea, A., \& Bolland, S. W. (2000). Target similarity effects: Support for the parallel distributed processing assumptions. Memory \& Cognition, 28, 798-811.

Just, M. A., \& CARPEnTER, P. A. (1992). A capacity theory of compre- hension: Individual differences in working memory. Psychological Review, 99, 122-149.

Kintsch, W. (1988). The role of knowledge in discourse comprehension: A construction-integrationmodel. Psychological Review, 95, 163182.

Lovett, M. C., Reder, L. M., \& Lebiere, C. (1999). Modeling working memory in a unified architecture: An ACT-R perspective. In A. Miyake $\&$ P. Shah (Eds.), Models of working memory: Mechanisms of active maintenance and executive control (pp. 135-182). Cambridge: Cambridge University Press.

MCGEOCH, J. A. (1942). The psychology of human learning. New York: Longmans, Green \& Co.

Nelson, D. L., \& Goodmon, L. B. (2002). Experiencing a word can prime its accessibility and its associative connections to related words. Memory \& Cognition, 30, 380-398.

Nelson, D. L., McEvoy, C. L., JAnczura, G. A., \& Xu, J. (1993). Implicit memory and inhibition. Journal of Memory \& Language, 32, 667-691.

Nelson, D. L., McEvoy, C. L., \& Schreiber, T. A. (1999). The University of South Florida Word Association, Rhyme and Fragment Norms [Database]. http://www.usf.edu/FreeAssociation/.

Nelson, D. L., McKinney, V. M., Gee, N. R., \& Janczura, G. A. (1998). Interpreting the influence of implicitly activated memories on recall and recognition. Psychological Review, 105, 299-324.

Nelson, D. L., \& Zhang, N. (2000). The ties that bind what is known to the recall of what is new. Psychonomic Bulletin \& Review, 7, 604617.

SCHNEIDER, W. (1999). Working memory in a multilevel hybrid connectionist control architecture (CAP2). In A. Miyake \& P. Shah (Eds.), Models of working memory: Mechanisms of active maintenance and executive control (pp.340-374). Cambridge: Cambridge University Press.

Sмiтн, S. M. (1979). Remembering in and out of context. Journal of Experimental Psychology: Human Learning \& Memory, 5, 460-471.

Thorndike, E. L. (1914). The psychology of learning. New York: Teachers College.

UNDERWOOD, B. J. (1965). False recognition produced by implicit verbal responses. Journal of Experimental Psychology, 70, 122-129.

\section{NOTE}

1. PIER2 assumes that two independent representations of the target are produced during the study experience. An explicit representation is produced as a result of episodic experience with the study word, and an implicit representation is produced during comprehension as a result of what is activated in long-term working memory. Thus far, only the implicit representation has been developed in model form (see the Appendix). 


\section{APPENDIX \\ A Computational Example Using PIER2's Equations}

1. Equation A1 (the target activation process) assumes that studying a word primes its representation and the representations of its strongest associates in long-term working memory. The initial self-strength of the target is assigned a nominal value of 1.00, and remaining values are taken from free-associationnorms (e.g., the probabilities that the target will produce Associates 1-3 are .50, .30, and .20, respectively, the probabilities that Associate 1 will produce the target and the target's Associate 2 are .30 and .10, respectively, and so forth). Equation A1 assumes that the activation strengths of the target and its associates increase as an additive function of the strength of preexisting links in the network. Targets with more and stronger links to, from, and among their associates are activated to higher levels than those with fewer and weaker links (see Table A1). Activation strength for the target and each associate is defined and computed as:

$$
\begin{aligned}
S\left(T_{i}\right)=\left[S(T, T)+\sum_{i=1}^{n} S\left(A_{i}, T\right)\right]+\sum_{j=1}^{n}\left[S\left(T, A_{j}\right)+\sum_{i=1}^{n} S\left(A_{i}, A_{j}\right)\right] \\
=(1.00+.30+.40)+[(.50+.30+.10)+(.30+.10)+(.20)]=3.20 \\
S\left(A_{i}\right)=\sum_{j=1}^{n}\left[S\left(T, A_{j}\right)+\sum_{i=1}^{n} S\left(A_{i}, A_{j}\right)\right]
\end{aligned}
$$

therefore

$$
S\left(A_{1}\right)=[(.50)+(.30+.10)]=.90 .
$$

2. Equation A2 (the cue-target intersection process) uses the activation levels of the target and its associates in computing the intersection between this information and that activated by the test cue. Forward cue-to-target links (F), backward target-to-cuelinks (B), shared associates (S), and mediators (M) all contribute to the signal strength of the target in relation to its cue. For example, to calculate Equation A2 with the target's Associate 1 as the cue, the results of Equation A1 for the target and its shared associates are folded into the top row of the retrieval matrix (see Table A2). For the test cue, normative values for free-association strength are entered into the bottom row of the matrix for forward-strength and shared associates. For mediated connections-for example, Associate 1-Mediator-Target-the mediator-to-target link is not primed during study. Only normative values are used for links in a mediated chain. The strength of target $T_{i}$ given test cue $Q_{j}$ is

$$
\begin{aligned}
S\left(Q_{j}, T_{i}\right)= & \sum_{k}^{n} S_{j k} S_{i k}+\sum_{k}^{n} S_{j k} S_{k i}=(.30 \times 3.20)+(1.00 \times .90) \\
& +(.10 \times .40)+(.15 \times .15)=1.92 .
\end{aligned}
$$

3. Equation A3 (target recovery) computes a signal-to-noise ratio that incorporates the strengths of competing associates activated by the target and by the test cue as noise elements. For example, Associate 3 is not produced as an associate of the test cue, so it is not a shared associate. On the assumption that it is also not a mediator, it competes with the target for recall. The computation shown in Table A3 assumes that the test cue produces two competitors $\left(\mathrm{C}_{1}, \mathrm{C}_{2}\right)$. Any associate of the target or test cue that does not contribute to strength

Table A1

Activation Matrix

\begin{tabular}{lcccc}
\hline & Target & Associate 1 & Associate 2 & Associate 3 \\
\hline Target & 1.00 & .50 & .30 & .20 \\
Associate 1 & .30 & - & .10 & \\
Associate 2 & .40 & .30 & - & - \\
Associate 3 & & .10 & .40 & .20 \\
Activation strength & 3.20 & .90 & & \\
\hline
\end{tabular}

Table A2

Retrieval Matrix

\begin{tabular}{ccccc}
\hline & Test Cue & \multicolumn{3}{c}{ Shared Associate $(\mathrm{S})$} \\
& Target & (Associate 1) & (Associate 2) & Mediator (M) \\
\hline Target & 3.20 & $.90(\mathrm{~B})$ & .40 & .15 \\
Test cue (Associate 1) & $.30(\mathrm{~F})$ & 1.00 & .10 & .15 \\
\hline
\end{tabular}


APPENDIX (Continued)

Table A3

Competition Matrix

\begin{tabular}{lcc}
\hline & $\mathrm{C}_{1}$ & $\mathrm{C}_{2}$ \\
\hline Target & .20 & - \\
Associate 1 & .30 & .15 \\
\hline
\end{tabular}

contributes to noise. Free-association norms are used to estimate the strengths of the competitors, and the summed strengths of all competitors determine the noise level.

The probability of sampling the target is the presence of noise is

$$
P_{s}\left(T_{i} / Q_{j}\right)=\frac{S\left(Q_{j}, T_{i}\right)}{S\left(Q_{j}, T_{i}\right)+\alpha \sum_{q}^{n} S\left(Q, A_{q}\right)+\beta \sum_{t}^{n} S\left(T, A_{t}\right)},
$$

where $\alpha, \beta=1.0$ in this example, $q=$ the competing associates of the test cue, and $t=$ the competing associates of the target. Hence, in the example:

$\operatorname{Ps}($ Target/Associate 1 as the test cue $)=192 \div[1.92+.20+.30+.15]=.75$.

4. Predictions. On nondisrupted tests, PIER2's equations predict that recall probability will be ordered as: $\mathrm{F}$ $+\mathrm{B}>\mathrm{F}>\mathrm{B}$. These predictions are easy to see in the computation for Equation A2. Net cue-target strength will be greater when both links are available than when only one link is available, because missing links are assigned values of zero. Furthermore, $\mathrm{F}$ generally will be greater than $\mathrm{B}$ because of the assumed target activation process, resulting in $(\mathrm{F} \times \mathrm{T})>(1.0 \times \mathrm{B})$. Similarly, for shared associates $(\mathrm{S})$ and mediators $(\mathrm{M})$, the model predicts a recall ordering of $S+M>S=M>$ control at fixed levels of $S$ and $M$ strength. On disrupted tests, as long as the probability of recovering the target is greater than the free-association baseline probability, the model predicts that the orderings obtained on the nondisrupted tests will be maintained. As access begins to approach this baseline, the difference between the cues will be reduced, and eventually $\mathrm{F}+\mathrm{B}=\mathrm{F}>\mathrm{B} . \mathrm{F}+\mathrm{B}$ and $\mathrm{F}$ cues will approach .13 in Experiment 1, which is the free-association probability that $\mathrm{F}$ will produce the target in the absence of study. B will approach zero because B-link cues do not produce their targets in free association. The exact form of the access loss function has not been worked out, but the model does produce qualitative predictions. These predictions can be confirmed by recomputing Equation A2 for each cue type (e.g., $\mathrm{F}+\mathrm{B}, \mathrm{F}, \mathrm{B}$ ) assuming that $100 \%, 60 \%$, and $40 \%$, respectively, of the activation values shown for Equation A1 are accessible. Such computations will assume that cue and target competitors will remain the same (see Equation A3), but this assumption is reasonable because cue and target set size are controlled in these experiments.

(Manuscript received May 31, 2002;

revision accepted for publication August 23, 2002.) 